\title{
Generation of Hulls Encompassing Neuronal Pathways Based on Tetrahedralization and 3D Alpha Shapes
}

\author{
Dorit Merhof $^{1,2}$, Martin Meister ${ }^{1}$, Ezgi Bingöl ${ }^{1}$, \\ Peter Hastreiter $^{1,2}$, Christopher Nimsky ${ }^{2,3}$, Günther Greiner ${ }^{1}$ \\ ${ }^{1}$ Computer Graphics Group, University of Erlangen-Nuremberg, Germany \\ ${ }^{2}$ Neurocenter, Dept. of Neurosurgery, University of Erlangen-Nuremberg, Germany \\ ${ }^{3}$ Dept. of Neurosurgery, University of Erlangen-Nuremberg, Germany \\ Email: dorit.merhof@informatik.uni-erlangen.de
}

\begin{abstract}
Diffusion tensor imaging provides information about structure and location of white matter tracts within the human brain which is of particular interest for neurosurgery. The reconstruction of neuronal structures from diffusion tensor data is commonly solved by tracking algorithms based on streamline propagation. These approaches generate streamline bundles that approximate the course of neuronal fibers. For medical application, a 3D representation of streamline bundles provides valuable information for pre-operative planning. However, for intra-operative visualization, surfaces wrapping eloquent structures are required for integration into the OR microscope. In order to provide hulls tightly encompassing the neuronal structures obtained from fiber tracking, we propose an approach based on tetrahedralization. This technique reuses the sampling points derived from fiber tracking and therefore provides precise hulls which serve as basis for intra-operative visualization.
\end{abstract}

\section{Introduction}

In recent years, diffusion tensor imaging (DTI) data has gained increasing interest due to its capability to reflect location and structure of fibrous tissue such as white matter in vivo. For this reason, DTI data is of high value in neurosurgery enhancing the information obtained from standard magnetic resonance imaging (MRI) data. For pre-operative planning as well as intraoperative visualization, tract systems such as the pyramidal tract, the optical tract or the corpus callosum are reconstructed.

Commonly accepted techniques for fiber tract reconstruction from DTI data are fiber tracking algorithms. Respective tracking results indicate the location of white matter tracts within the human brain. In the context of

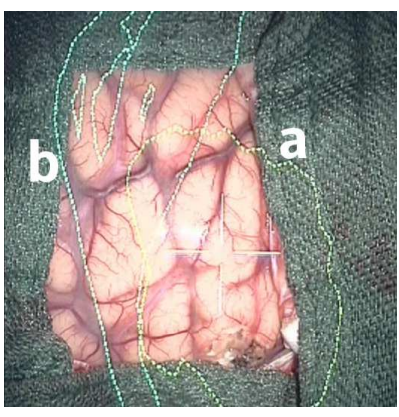

Fig. 1. Microscope view. Tumor (a) and pyramidal tract (b) in close neighborhood. neurosurgery, fiber bundles obtained from fiber tracking provide valuable information for diagnosis and therapy planning. However, for intra-operative visualization of fiber tract data, hulls tightly wrapping these structures are required. During surgery, the 
boundary curves of the hulls are displayed in the focus plane of the OR microscope and provide a direct relation between tumor tissue and neuronal structures (Figure 1).

A first approach for wrapping fiber tracts [1] computes the centerline of the fiber bundle. In a second step, the center line is sampled equidistantly and planes perpendicular to the center line are considered. For each plane, the intersecting points of all fibers with the plane are computed. Finally, an ellipse encompassing all intersection points is defined for each plane and the ellipses of subsequent planes are connected using a triangular mesh. This approach provides hulls that fit the underlying fiber structure. However, the technique does not take into account branching fibers, requiring a splitting center line or a more sophisticated solution for defining ellipses and connecting them appropriately. In addition to that, the technique is restricted to elongated tract systems, where a centerline is well defined. For fiber tracts such as the corpus callosum encompassing fibers with significantly varying course and direction, the approach will fail.

For this reason, we present a novel hull algorithm overcoming these drawbacks. In order to provide precise hulls, the technique takes advantage of the sampling points of the tracked fibers to guarantee high precision. In a first step, a tetrahedral mesh is constructed from the sampling points based on 3D Delaunay tetrahedralization. Since the tetrahedralization process results in the convex hull of the fiber tract, a variation of the 3D alpha shape algorithm has to be applied. As a result, the triangles on the surface of the remaining tetrahedral mesh describe a hull precisely encompassing the fiber tract.

\section{Material}

All datasets used in this work were measured using a Siemens MR Magnetom Sonata Maestro Class 1.5 Tesla scanner. The specifications of the gradient system were a field strength of up to $40 \mathrm{mT} / \mathrm{m}$ (effective $69 \mathrm{mT} / \mathrm{m}$ ) and a slew rate of up to $200 \mathrm{~T} / \mathrm{m} / \mathrm{s}$ (effective $346 \mathrm{~T} / \mathrm{m} / \mathrm{s}$ ) for clinical application.

DTI datasets were acquired using a field of view of $240 \mathrm{~mm}$ resulting in a voxel size of $1.875 \times 1.875 \times 1.9 \mathrm{~mm}^{3}$. For each of the six diffusion weighted datasets (gradient directions $( \pm 1,1,0),( \pm 1,0,1)$ and $(0,1, \pm 1))$ and the reference dataset, sixty slices with no intersection gap and an acquisition matrix of $128 \times 128$ pixels were measured.

\section{Methods}

In this section, the steps for hull generation, comprising fiber tracking (Section 3.1), tetrahedralization (Section 3.2) and 3D alpha shapes (Section 3.3), are described.

\subsection{Fiber tracking}

In a first step, fiber tracts were computed using a streamline-based tracking approach incorporating trilinear tensor interpolation and fourth order Runge-Kutta integration [2]. Fractional anisotropy was used as termination threshold for fiber propagation. Single tract systems were obtained by incorporating ROIs (regions of interest) defined by a 
medical expert into the tracking process. As a result, fiber tracts corresponding to specific function such as the pyramidal tract (motor), the optical tract (vision) and the corpus callosum (connection between the two hemispheres) are obtained.

\subsection{Tetrahedralization}

The point set comprising the sampling points of all fibers within the fiber tract is then used as input for the tetrahedralization algorithm. For the reconstruction of a tetrahedral mesh based on this point set, a 3D Delaunay [3] approach is applied. For points in general position, i.e. no geometric test is ambiguous, this tetrahedralization is uniquely defined and decomposes the convex hull of the point set into tetrahedra [3]. The tetrahedralization of a point set fulfills the 3D Delaunay criterion, if each sphere defined by the four points of a tetrahedron contains in its interior no other point of the point set. For implementation purposes, the vtkDelaunay3D class of the Visualization ToolKit (VTK) [4] was used. In Figure 2 (left), the surface of the initial set of tetrahedra derived from vtk's 3D Delaunay tetrahedralization is shown.

\subsection{D Alpha Shapes}

The output of the 3D Delaunay algorithm is a tetrahedral mesh filling the convex hull of the point set with volume elements. In order to obtain the subset of tetrahedra tightly enclosing the fiber tract, outer tetrahedra have to be removed in an iterative process. For this purpose, a variation of the 3D alpha shape algorithm is applied. The concept of alpha shapes [5] is a generalization of the convex hull, formalizing the intuitive notion of 'shape' for spatial point set data. Depending on the alpha value, which is a real number greater than zero, the alpha shape of an object encompasses only those tetrahedra with smaller or equal circumsphere than a sphere with diameter alpha. For sufficiently large alpha, the alpha shape is identical to the convex hull which is the original tetrahedral mesh. For decreasing values of alpha, approaching the step size used for fiber tracking, the alpha shape shrinks and gradually reveals the shape of the fiber tract.
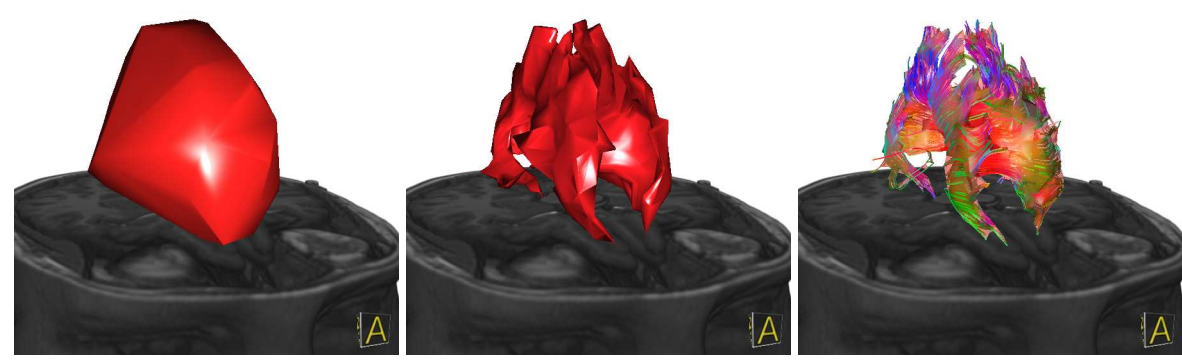

Fig. 2. 3D Delaunay tetrahedralization based on sampling points of a tract system (corpus callosum) results in the convex hull (left). Subset of tetrahedra obtained with 3D alpha shapes for an alpha value of 10 (middle) and semi-transparent hull for an alpha value of 5 displayed with tracked fibers (right). 
When applying the alpha shape concept to the tetrahedral meshes obtained from 3D Delaunay tetrahedralization, holes may occur due to removal of inner tetrahedra. For this reason, a variation of the 3D alpha shape algorithm is used, where tetrahedra are only removed according to the alpha criterion, if they are on the surface of the current tetrahedral mesh. This is implemented by setting an alpha-flag for all tetrahedra which should be removed according to the alpha value, and a boundary-flag for all tetrahedra on the surface. In an iterative procedure, surface tetrahedra with valid alpha-flag are removed, and the boundary-flag of their neighbor elements is set. Sweeping through the tetrahedra data structure continues, as long as tetrahedra for removal are found.

After applying the 3D alpha shape algorithm, a tetrahedral mesh remains which exactly corresponds to the intuitive shape of the fiber tract. The triangular hull mesh is constructed from the outer faces of the surface tetrahedra.

\section{Results and Discussion}

The novel technique for precise hull generation was applied to different tract systems, namely the pyramidal tract (motor), the optical tract (vision) and the corpus callosum (connection between the two hemispheres). For all tract systems, the algorithm succeeded to generate precise hulls following the shape of the fiber bundle, as shown in Figure 2 and 3.

In comparison to the initial approach for wrapping fibers [1], the presented technique provides higher precision which is an essential feature for the intended application. This is due to the fact, that points originating from fiber tracking are directly used

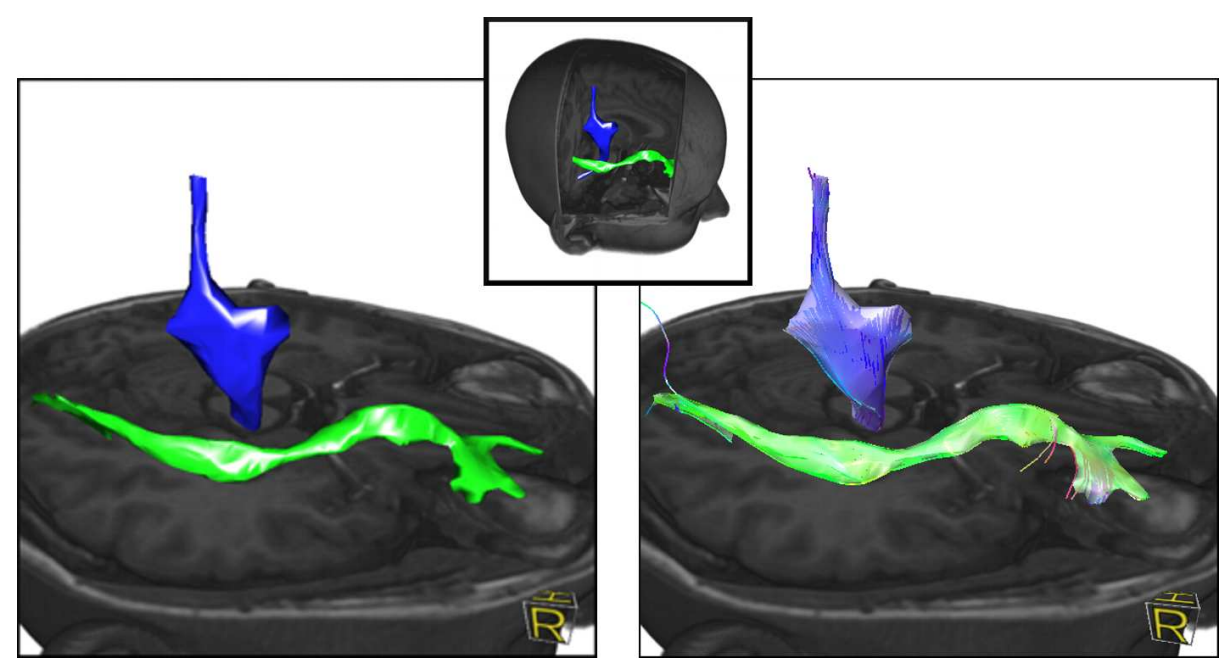

Fig. 3. Hulls obtained from tetrahedralization and 3D alpha shapes for an alpha value of 5 for the pyramidal (blue) and the optic tract (green). Semi-transparent visualization of the hulls in combination with the fibers (right) shows the high precision of the approach. 
for tetrahedralization and remain after application of 3D alpha shapes. Additionally, the algorithm is also able to wrap branching fiber tracts or tract systems with diverging fiber directions. With respect to computing times, the algorithm is more time consuming due to the reconstruction of the tetrahedral mesh. For a fiber tract comprising 7443/ 20139 / 70352 points (pyramidal tract / optical tract / corpus callosum), the 3D Delaunay tetrahedralization requires 2.3 / 8.5 / 80.2 seconds (on a PC equipped with a P4 3.0 GHz and $2 \mathrm{~GB}$ RAM). The remaining computations to eliminate unneeded tetrahedra (Section 3.3) are in the range of few seconds. Computing times could be lowered by a coarser sampling of the fibers, or by advanced tracking strategies controlling the density of single fibers, both resulting in a smaller point set. However, for the purpose of preoperative planning and intra-operative visualization, the major goal for hull generation are accuracy aspects, not necessarily minimal computing times.

\section{Conclusion}

We presented a novel method for computing hulls encompassing neuronal pathways. As an advantage over existing techniques, the approach is capable to wrap tract systems of arbitrary shape such as branching or winding fiber tracts. In addition to that, the resulting hulls tightly fit the underlying fiber structure since the hull mesh is composed from sampling points derived by fiber tracking. Overall, the presented technique is able to wrap fiber tracts of any shape, and at the same time provides maximum wrapping precision. For medical application, this is of high value in order to obtain a precise visualization denoting the localization of white matter tracts.

\section{Acknowledgments}

This work was supported by the Deutsche Forschungsgemeinschaft in the context of SFB 603, Project C9 and the Graduate Research Center "3D Image Analysis and Synthesis". We thank Frank Enders for contributions to the visualization framework.

\section{References}

1. Enders, F., Sauber, N., Merhof, D., Hastreiter, P., Nimsky, C., Stamminger, M.: Visualization of white matter tracts with wrapped streamlines. In: Proc. IEEE Visualization. (2005) 51-58

2. Merhof, D., Enders, F., Vega, F., Hastreiter, P., Nimsky, C., Stamminger, M.: Integrated visualization of diffusion tensor fiber tracts and anatomical data. In: Proc. Simulation and Visualization. (2005) 153-164

3. Delaunay, B.: Sur la sphère vide. Bulletin of Academy of Sciences of the USSR (VII) (1934) 793-800

4. Schroeder, W., Martin, K., Lorensen, B.: The Visualisation ToolKit. Kitware (2002), URL: http://www.vtk.org.

5. Edelsbrunner, H., Mücke, E.: Three-dimensional alpha shapes. ACM Transactions on Graphics 13 (1994) 43-72 